\title{
Integrated medical school ultrasound: development of an ultrasound vertical curriculum
}

\author{
David P Bahner $^{1 *}$, Eric J Adkins ${ }^{1,2}$, Daralee Hughes ${ }^{1}$, Michael Barrie ${ }^{1}$, Creagh T Boulger ${ }^{1}$ and Nelson A Royall ${ }^{3,4}$
}

\begin{abstract}
Background: Physician-performed focused ultrasonography is a rapidly growing field with numerous clinical applications. Focused ultrasound is a clinically useful tool with relevant applications across most specialties. Ultrasound technology has outpaced the education, necessitating an early introduction to the technology within the medical education system. There are many challenges to integrating ultrasound into medical education including identifying appropriately trained faculty, access to adequate resources, and appropriate integration into existing medical education curricula. As focused ultrasonography increasingly penetrates academic and community practices, access to ultrasound equipment and trained faculty is improving. However, there has remained the major challenge of determining at which level is integrating ultrasound training within the medical training paradigm most appropriate.

Methods: The Ohio State University College of Medicine has developed a novel vertical curriculum for focused ultrasonography which is concordant with the 4-year medical school curriculum. Given current evidenced-based practices, a curriculum was developed which provides medical students an exposure in focused ultrasonography. The curriculum utilizes focused ultrasonography as a teaching aid for students to gain a more thorough understanding of basic and clinical science within the medical school curriculum. The objectives of the course are to develop student understanding in indications for use, acquisition of images, interpretation of an ultrasound examination, and appropriate decision-making of ultrasound findings.
\end{abstract}

Results: Preliminary data indicate that a vertical ultrasound curriculum is a feasible and effective means of teaching focused ultrasonography. The foreseeable limitations include faculty skill level and training, initial cost of equipment, and incorporating additional information into an already saturated medical school curriculum.

Conclusions: Focused ultrasonography is an evolving concept in medicine. It has been shown to improve education and patient care. The indications for and implementation of focused ultrasound is rapidly expanding in all levels of medicine. The ideal method for teaching ultrasound has yet to be established. The vertical curriculum in ultrasound at The Ohio State University College of Medicine is a novel evidenced-based training regimen at the medical school level which integrates ultrasound training into medical education and serves as a model for future integrated ultrasound curricula.

Keywords: Curriculum; Focused ultrasound; Medical education; Ultrasonography; Undergraduate medical education

\footnotetext{
* Correspondence: david.bahner@osumc.edu

${ }^{1}$ Department of Emergency Medicine, The Ohio State University College of Medicine, 750 Prior Hall, 376 W 10th Avenue, Columbus, OH 43210, USA Full list of author information is available at the end of the article
}

\section{国 Springer}

(c) 2013 Bahner et al.; licensee Springer. This is an Open Access article distributed under the terms of the Creative Commons Attribution License (http://creativecommons.org/licenses/by/2.0), which permits unrestricted use, distribution, and reproduction in any medium, provided the original work is properly cited. 


\section{Background}

Physician-performed focused ultrasonography represents a novel approach to patient evaluation and treatment in the acute and chronic settings [1]. Ultrasound has been utilized for decades by traditional imaging specialists (radiologists, cardiologists, and obstetric-gynecologists) within the comprehensive ultrasound paradigm, in which a sonographer performs the examination followed by a physician evaluating the images. The recent development of portable and handheld ultrasound machines has led to a rapid growth in an emerging field called focused ultrasonography [2]. Unlike traditional imaging modalities, focused ultrasound is available as a bedside tool which can be both performed and interpreted instantly by the physician. Focused ultrasound has been demonstrated to improve rapid diagnosis, clinical management, and procedural performance in multiple studies [1]. As shown in Table 1, the potential uses for focused ultrasonography are broad and cross many specialties.

In recognition of the potential role of focused ultrasound within clinical practice, the American Medical Association (AMA) has affirmed that bedside focused ultrasound is within the scope of practice of appropriately trained physicians [3,4]. Governing bodies for physician specialties have also begun to define the scope of practice for physician-performed focused ultrasonography. For example, the American College of Emergency
Physicians (ACEP) defined the use of ultrasonography in trauma, intrauterine pregnancy, abdominal aortic aneurysm, cardiac, biliary, urinary tract, venous thromboembolism, soft tissue, musculoskeletal, thoracic, and ocular evaluation and procedural guidance to be within the emergency physician's scope of practice $[5,6]$. Moreover, billing and reimbursement for focused ultrasound has been supported through the AMA Physicians' Current Procedural Terminology (CPT) 2011 codes which include CPT code modifiers for focused ultrasonography [7]. Despite supporting evidence and administrative policies for physician-performed focused ultrasonography, an educational strategy and widely accepted guidelines for competency for focused ultrasonography have yet to be established.

\section{Integration of ultrasound into medical education}

A central issue in training physicians in ultrasonography lies in locating the time and funding for training programs. Initial attending-level programs provided evidence for the use of physician-performed focused ultrasonography, and participants demonstrated rapid skill acquisition and improved interpretation skills following these courses [8]. These courses however are generally limited to hospital-based programs or subspecialty-based educational forums which are further limited by single-session time constraints and are unable to provide longitudinal training and ongoing skill assessment.

Table 1 Currently described applications for physician-performed focused ultrasonography defined by physician specialty

\begin{tabular}{|c|c|}
\hline Specialty & Applications \\
\hline Internal medicine & Hypotension evaluation and treatment, procedural guidance \\
\hline Cardiology & Cardiac evaluation, procedural guidance \\
\hline Gastroenterology & Liver evaluation, intestinal obstruction evaluation, procedural guidance \\
\hline Infectious disease & Soft tissue infection evaluation, procedural guidance \\
\hline Nephrology & Renal evaluation, procedural guidance \\
\hline Pulmonology & Pleural effusion evaluation, atelectasis evaluation, pneumothorax evaluation, procedural guidance \\
\hline Rheumatology & Joint and tendon evaluation, procedural guidance \\
\hline Pediatrics & Developmental abnormality evaluation, abdominal pain evaluation, procedural guidance \\
\hline Emergency medicine & $\begin{array}{l}\text { Hypotension evaluation, intrauterine pregnancy evaluation, abdominal aortic aneurysm evaluation, cardiac } \\
\text { evaluation, biliary tree evaluation, urinary tract evaluation, venous thromboembolism evaluation, soft tissue } \\
\text { and musculoskeletal evaluation and treatment, lung evaluation, ocular evaluation, procedural guidance }\end{array}$ \\
\hline Obstetrics-gynecology & Intrauterine pregnancy evaluation, fetal development evaluation, ovary evaluation \\
\hline Surgery & Trauma evaluation, breast and thyroid evaluation, vascular evaluation, intraoperative evaluation, procedural guidance \\
\hline Anesthesia & Intraoperative monitoring, procedural guidance \\
\hline Critical care & $\begin{array}{l}\text { Hypotension evaluation, intravascular volume evaluation and treatment, cardiac evaluation, lung evaluation, } \\
\text { intracranial pressure monitoring, procedural guidance }\end{array}$ \\
\hline Neurology & Transcranial evaluation \\
\hline Neurosurgery & Transcranial and intracranial evaluation, intraoperative guidance \\
\hline Orthopedic surgery & Joint and tendon evaluation and treatment, procedural guidance \\
\hline Urology & Urinary tract evaluation \\
\hline Plastic surgery & Intraoperative evaluation \\
\hline
\end{tabular}


Currently focused ultrasonography is a required component of Emergency Medicine, Obstetrics-Gynecology, Internal Medicine, and Radiology and recommended for General Surgery and Anesthesiology residency training programs [9-11]. Traditional training at the resident level was first pioneered through Radiology and ObstetricsGynecology, although these programs were designed to teach comprehensive ultrasonography within their respective areas of interest [12]. Other specialties such as General Surgery have developed resident training in focused ultrasonography, in particular for evaluation and management of traumatic injuries [13]. The experiences gained in these programs vary greatly, and to date many general surgeons may rely on Emergency Medicine or Radiology physicians to perform a Focused Assessment with Sonography in Trauma (FAST) examination. Perhaps the most advanced training programs in focused ultrasonography have come from Emergency Medicine, where residents are recommended to develop a proficiency in 11 different ultrasound foci $[9,14]$. However, these recommendations have not been accompanied by curricula, competency assessment, or a method to standardize the quality and quantity of focused ultrasound exposure across programs. This variability between specialties and even residency programs demonstrates a need for a common foundational curriculum.

\section{Role of ultrasound within medical school}

Focused ultrasonography has the potential to be both an educational adjunct and a clinical tool for medical students [15-29]. Early analyses demonstrated that in small cohorts, medical students were able to develop the psychomotor and interpretative skills required for effective focused ultrasonography [17,20,22,25,26,29]. For example, a study at Wayne State University showed that first-year medical students were able to successfully utilize portable ultrasound to differentiate sonographic objects following six 90-min sessions covering abdominal, cardiovascular, genitourinary, and musculoskeletal applications [29]. Additional efforts have demonstrated that focused ultrasonography may be useful as an educational aid in teaching anatomy to medical students $[15,16,18,19,27]$. A study from the Mayo Clinic demonstrated that fourth-year medical students who used focused echocardiography to aid in the understanding of cardiovascular anatomy had high satisfaction rates [15]. Further, the use of focused ultrasonography among medical school students has been shown to potentially aid in the development of physical examination skill acquisition $[19,28]$. In a study from the University of Chicago, fourth-year medical students used focused echocardiography in cardiac evaluation with subsequent improved detection of cardiac conditions and higher accuracy in cardiac auscultation skills [19].
Medical students have been shown to adequately develop focused ultrasound skills for clinical practice through multiple small studies [21,28,30]. Despite the demonstrated benefits, there have been only limited efforts to develop a comprehensive and organized curriculum utilizing focused ultrasonography in medical school $[28,31]$. The need for a vertical curriculum which incorporates ultrasound education into an existing medical school curriculum was the basis for development of an ultrasound vertical curriculum at The Ohio State University College of Medicine (OSU COM).

\section{Methods}

The purpose of the ultrasound vertical curriculum was to develop medical student proficiency in focused ultrasonography that would allow graduates to (1) identify appropriate uses of focused ultrasonography, (2) acquire real-time ultrasound images and video, (3) interpret ultrasound findings, and (4) utilize ultrasound findings in clinical decision-making. A curriculum was developed based upon three learner theory model core components: cognitive, behavioral, and constructive. Due to curriculum time constraints, the decision was made to emphasize cognitive and behavioral learning components within mandatory courses and include constructive components predominately within the elective or selective courses. Table 2 shows the integration of vertical ultrasound curriculum components within the current medical school curriculum.

\section{Objectives of an integrated curriculum}

The curriculum was constructed to accomplish objectives within focused ultrasonography which are complimentary to the larger medical school curriculum. Given the predominance of basic science and clinical skills taught during the first 2 years of the curriculum, emphasis on ultrasound basic science and image acquisition was chosen. Within the clinical years, an emphasis on ultrasound indications and interpretation was selected.

In the first medical school year, the curriculum covered basic ultrasound physics including ultrasound wave production, propagation within media, and image production. Also, students learned how to utilize a portable ultrasound machine to acquire ultrasound images and identify basic anatomy given respective acoustic properties. In the second medical school year, students learned advanced ultrasound physics including variations in ultrasound transducer wave production, artifact production, and thermo-mechanical heat production with a basic understanding of associated relative risks. Following the second year, students were able to understand image acquisition limitations and methods to overcome challenges to image acquisition as well as utilize ultrasound during basic procedural guidance. In the third medical school year, 
Table 2 An overview of four-year integrated vertical ultrasound curriculum developed at The Ohio State University College

\begin{tabular}{|c|c|c|c|c|}
\hline \multirow{3}{*}{$\frac{\text { Medical school year }}{\text { Medical School Year } 1}$} & \multicolumn{4}{|c|}{ Components } \\
\hline & \multirow[t]{3}{*}{3 Months } & \multirow{3}{*}{$\begin{array}{l}\text { Gross Anatomy and } \\
\text { Laboratory }\end{array}$} & \multicolumn{2}{|l|}{ Musculoskeletal Anatomy Ultrasound } \\
\hline & & & \multicolumn{2}{|c|}{ Thorax, Abdomen and Pelvis Anatomy Ultrasound } \\
\hline & & & \multicolumn{2}{|l|}{ Head and Neck Anatomy Ultrasound } \\
\hline & 7 Months & Basic Science Curriculum & \multicolumn{2}{|c|}{ Introduction to Focused Ultrasound Elective ${ }^{a}$} \\
\hline & 2 Months & Vacation & & \\
\hline \multirow[t]{3}{*}{ Medical School Year 2} & 8 Months & Basic Science Curriculum & $\begin{array}{l}\text { Basic Course in Focused Ultrasound } \\
\text { Protocols Elective }^{\mathrm{a}}\end{array}$ & \multirow[t]{2}{*}{ Ultrasound Model Pool Elective } \\
\hline & 2 Weeks & Introduction to Clinical Medicine & Ultrasound-Guided Vascular Access & \\
\hline & 2 Months & Vacation & & \\
\hline \multirow[t]{7}{*}{ Medical School Year $3^{\mathrm{b}}$} & 8 Weeks & Internal Medicine & \multirow{6}{*}{\multicolumn{2}{|c|}{ Specialty-Based Hands-On Ultrasound Experience }} \\
\hline & 8 Weeks & Pediatrics & & \\
\hline & 8 Weeks & Family Medicine & & \\
\hline & 8 Weeks & General Surgery & & \\
\hline & 8 Weeks & Neurology/Psychiatry & & \\
\hline & 6 Weeks & Obstetrics-Gynecology & & \\
\hline & 2 Weeks & Clinical Topics & \multicolumn{2}{|l|}{ Core Focused Ultrasound Protocols } \\
\hline \multirow[t]{5}{*}{ Medical School Year 4} & 1 Month & Emergency Medicine Rotation & Emergency Focused Ultrasound & \multirow{5}{*}{$\begin{array}{l}\text { Advanced Course in } \\
\text { Focused Ultrasound Elective }\end{array}$} \\
\hline & 1 Month & Ambulatory Medicine Rotation & \multirow{4}{*}{$\begin{array}{l}\text { Specialty-Based Hands-On Ultrasound } \\
\text { Experience }\end{array}$} & \\
\hline & 1 Month & Chronic Care Rotation & & \\
\hline & 1 Month & Sub-Internship Rotation & & \\
\hline & 7 Months & Elective Rotations & & \\
\hline
\end{tabular}

${ }^{\mathrm{a}}$ An elective course which is not mandatory for students; ${ }^{\mathrm{b}}$ only selected specialties are participated.

students learned to identify general indications for specific focused ultrasound examinations and the proper timeline in the clinical scenario for their use as well as gaining a deeper understanding of ultrasound for procedural guidance. In the fourth medical school year, students were able to identify limitations of focused ultrasonography and identify the sensitivity and specificity for focused ultrasonography for basic ultrasound protocols as well as understand risks and benefits associated with focused ultrasonography in clinical scenarios. Students were able to utilize focused ultrasonography at least within the scope of practice of their chosen specialty prior to graduation.

\section{Facilities and faculty}

The OSU COM is a single-campus university-based college with a university-based medical center on campus. The OSU COM is a 4-year medical school with 2,779 full-time faculty and approximately 863 students. The college curriculum is a traditional structure with two preclinical years of basic science and integrated clinical exposures followed by two clinical years. The preclinical course structure is an organ-based structure and contains longitudinal clinical mentorship for instruction. The clinical years consist of month-long rotations with mandatory rotations in Family Medicine, Internal Medicine, Pediatrics, Surgery, Obstetrics-Gynecology,
Neurology, and Psychiatry during the third year and selective rotations throughout the third year with a mandatory fourth-year Emergency Medicine rotation. The medical center consists of a 1,177-bed hospital system with 1,158 full-time clinical faculty in addition to residency and fellow training programs. The medical center contains portable ultrasound machines available for physician use within the Emergency Department, Surgical and Medical Intensive Care Units, Radiology Department, and medical and surgical patient floors.

The OSU COM contains a staffed simulation and training center (OSU COM Clinical Skills and Education Assessment Center) containing lecture rooms, simulation labs, and clinical rooms shared by the college and medical center. The clinical skill center simulation equipment includes ultrasound simulation models such as vascular phantoms for ultrasound-guided vascular access and pelvic phantoms for obstetrics and gynecology ultrasound examinations. Additionally, the center contains a dedicated ultrasound practical scanning center with four portable ultrasound machines with image and video archival systems.

\section{Curriculum description Medical school year 1}

Numerous gross anatomy adjuncts exist to aid understanding anatomical relationships and serve as an introduction 
to image comprehension. Unlike computed tomography, magnetic resonance imaging, or X-ray, ultrasound allows for hands-on exploration of anatomy. Medical students are provided 2-h-long lectures on basic ultrasound principles. The lectures review ultrasound wave production, propagation in tissue, and image production in addition to portable ultrasound machine knobology, transducers, and image acquisition techniques. Students participate in 1-h supervised hands-on sessions during each anatomy region. Students obtain ultrasound images of all required anatomical relationships for the respective anatomy region. Sessions are led by the ultrasound faculty and an anatomy professor. Individual guidance is provided by fourth-year medical students, and student volunteers serve as live models. Table 3 shows the anatomical structures and regions covered in the course. The practical anatomy examination for each anatomical region includes anatomy identification questions using ultrasound images and video.

After gross anatomy, a supplemental course is available for approximately 80 students to increase hands-on time and improve basic ultrasound education. The course utilizes the evaluation of a hypotensive patient with focused ultrasound as the main construct, with the established Trinity protocol as the primary assessment method [32]. Monthly lectures and hands-on sessions review the basic concepts covered during the gross anatomy ultrasound course and further explain concepts such as ultrasound wave production, propagation in media such as tissue, air, or fluid, and thermo-mechanical effects. The lectures explain the I-AIM model (indication, acquisition, interpretation, and medical decision-making) to students as a guide for utilizing focused ultrasonography [33]. Students are taught to recognize ultrasonographic findings such as pericardial effusions, cardiac motility disorders, abdominal free fluid, and aortic aneurysms. A post-course examination consisting of a practical examination on live student models and a written image identification examination is held to assess proficiency.

\section{Medical school year 2}

In preparation for the transition to clinical applications of preclinical training, medical students at OSU COM are exposed to a 2-week hands-on clinical skill review session in conjunction with a clinical reasoning course. An introduction to ultrasound-guided and ultrasoundassisted procedures is provided during the course by performing ultrasound-guided long- and short-axis peripheral and central vascular cannulation. Students are required to successfully differentiate venous from arterial vasculature using B-mode, compression, Doppler, and color ultrasonography. Online lectures through the EMSono site, an online learning management website, provide students with introductory didactics on basics
Table 3 Anatomical structures acquired and identified on live models during the ultrasound component of gross anatomy

\begin{tabular}{|c|c|}
\hline $\begin{array}{l}\text { Anatomical } \\
\text { region }\end{array}$ & Anatomical structure \\
\hline \multirow[t]{18}{*}{$\begin{array}{l}\text { Thorax and } \\
\text { abdomen }\end{array}$} & $\begin{array}{l}\text { Cardiac chambers (right and left ventricles, } \\
\text { right and left atria) }\end{array}$ \\
\hline & Pericardium \\
\hline & $\begin{array}{l}\text { Inferior vena cava (intra-abdominal and } \\
\text { intrathoracic) }\end{array}$ \\
\hline & Diaphragmatic caval hiatus \\
\hline & Cavo-atrial junction \\
\hline & Main portal vein \\
\hline & Intrahepatic portal veins (right and left) \\
\hline & Hepatic veins (right, middle, and left) \\
\hline & Gallbladder \\
\hline & Kidney (right and left) \\
\hline & Spleen \\
\hline & Hepatorenal recess (Morrison's pouch) \\
\hline & Aorta (intra-abdominal) \\
\hline & $\begin{array}{l}\text { Celiac trunk, splenic artery, common hepatic artery, } \\
\text { left gastric artery }\end{array}$ \\
\hline & $\underline{\text { Superior mesenteric artery }}$ \\
\hline & Inferior mesenteric artery \\
\hline & Renal artery (right and left) \\
\hline & Common iliac arteries (right and left) \\
\hline \multirow[t]{7}{*}{ Upper extremities } & Supraspinatus tendon \\
\hline & Subscapularis tendon \\
\hline & Biceps brachii tendon \\
\hline & Flexor digitorum profundus \\
\hline & Flexor digitorum superficialis \\
\hline & Flexor pollicis longus \\
\hline & Median nerve \\
\hline \multirow[t]{3}{*}{ Lower extremities } & Quadriceps muscle belly and central tendon \\
\hline & Patella \\
\hline & Patellar ligament \\
\hline \multirow[t]{6}{*}{ Head and neck } & Trachea \\
\hline & Thyroid gland \\
\hline & Thyroid cartilage \\
\hline & Internal jugular vein \\
\hline & Common carotid artery \\
\hline & Sternocleidomastoid \\
\hline
\end{tabular}

in ultrasound as an adjunct to hands-on training [34]. Post-didactic lecture quizzes are provided to ensure adequate understanding prior to completion of the hands-on sessions. A 1-h review of the procedural indications for ultrasound guidance and assistance as well as potential challenges in use such as image artifact production, site contamination, and equipment requirements is provided. 
In coordination with organ-based topics during the second pre-clinical medical school year, an elective course teaching focused ultrasound examination of relevant organ systems is available for approximately 40 students. The primary objectives of the longitudinal course are to provide increased exposure and review of basic ultrasound principles, additional focused ultrasound protocols, and hands-on experience with portable ultrasound machines. Basic ultrasound principles are presented, including machine operation such as patient identification, image orientation and labeling standards, and production of images and video using secondary and tertiary functions such as color or power Doppler, measurements, and labels. Ultrasound protocols and organ systems covered through the course are shown in Table 4. Additional required interactive online lectures are provided through the online resource EMSono, reviewing the topics covered in didactic lectures. Students record a log of ultrasound images and video supporting each ultrasound protocol completed and must perform at least five ultrasound examinations in each focus to meet a minimum of 25 examinations. Participation as an ultrasound model is an additional educational component used for the course to expose students to patient-focused issues such as patient positioning, probe pressure limitations, and anatomical limitations. Students were required to complete at least 10 hours of live ultrasound modeling as part of the course.

\section{Medical school year 3}

In the mandatory core clinical rotations during the third medical school year, OSU COM students complete an adjunct course focused on enhancing their understanding of ultrasound indications and image interpretation. The third year course consists of online interactive lectures, didactic lectures, and hands-on sessions. Previously described ultrasound protocols include the Trinity hypotensive examination, FAST, cardiac evaluation during ACLS, transvaginal pregnancy evaluation, and ultrasound-guided vascular access. Students complete an online interactive lecture through the EMSono resource prior to the course. A 2-h didactic lecture covers specific indications for focused ultrasonography and ultrasound image interpretation techniques and sample images [34]. Hands-on sessions then have students demonstrate focused ultrasonography in the required protocols, with an emphasis on evaluation of a clinical scenario and ultrasound image and video interpretation.

\section{Medical school year 4}

In conjunction with the mandatory Emergency Medicine rotation, fourth-year students are taught to develop practice management algorithms which incorporate focused ultrasonography as part of evidenced-based practices.
Table 4 Organ-systems and focused ultrasound protocols covered during elective longitudinal ultrasound course for second-year medical students

\begin{tabular}{|c|c|c|}
\hline Topic & $\begin{array}{l}\text { Focused ultrasound } \\
\text { protocol }\end{array}$ & Ultrasound images/video \\
\hline \multirow[t]{10}{*}{ Abdomen } & \multirow[t]{4}{*}{ FAST } & Perihepatic \\
\hline & & Perisplenic \\
\hline & & Pericardial (subxiphoid) \\
\hline & & Recto-uterine/recto-vesical \\
\hline & \multirow{4}{*}{$\begin{array}{l}\text { Abdominal aortic } \\
\text { aneurysm }\end{array}$} & Celiac trunk \\
\hline & & Superior mesenteric artery \\
\hline & & Renal arteries \\
\hline & & Common iliac arteries \\
\hline & \multirow{2}{*}{$\begin{array}{l}\text { Inferior vena cava } \\
\text { collapsibility index }\end{array}$} & Suprahepatic IVC during expiration \\
\hline & & Suprahepatic IVC during inspiration \\
\hline \multirow[t]{6}{*}{ Thoracic } & \multirow[t]{2}{*}{ Pneumothorax } & Pleural sliding \\
\hline & & M-mode pleural sliding \\
\hline & \multirow[t]{4}{*}{ Cardiac } & Parasternal long axis \\
\hline & & $\begin{array}{l}\text { Parasternal short axis (ventricle } \\
\text { apex, middle, and base) }\end{array}$ \\
\hline & & Apical \\
\hline & & Subxiphoid \\
\hline \multirow[t]{3}{*}{ Procedural } & \multirow[t]{3}{*}{ Vascular access } & Differentiation of artery and vein \\
\hline & & Needle vessel entry in short axis \\
\hline & & Needle vessel entry in long axis \\
\hline
\end{tabular}

Students complete online lectures in focused ultrasonography using the EMSono resource prior to the initial focused ultrasound session [34]. A 2-h didactic lecture on evidenced-based evaluation of a hypotensive patient is given, which includes a review of focused ultrasonography principles from the previous coursework such as ultrasound indications, acquisition, and interpretation. Students then complete a faculty-led hands-on session where students demonstrate an evaluation of a hypotensive patient, including the previously described Trinity hypotensive protocol, transvaginal evaluation for pregnancy, and placement of central venous catheters under ultrasound guidance. As part of the rotation, students demonstrate focused ultrasound examinations on Emergency Department patients in appropriate clinical scenarios under faculty guidance, consistent with the current ACEP ultrasound scope of practice guidelines [6].

Students entering specialties with demonstrated focused ultrasonography applications are able to enroll in an elective course in advanced ultrasound training. The previously described course holds monthly didactic lectures, hands-on sessions, and journal club sessions on a specific focused ultrasound topic. Enrollment in the course is limited to approximately 40 students per year. Didactic sessions are 2-h lectures from specialty-specific 
faculty discussing the indications, contraindications, acquisition techniques, and examination interpretation and management implications for the topic. Hands-on sessions are proctored by the ultrasound faculty with assistance from monthly faculty using volunteer students for practice scanning models. Journal club sessions use faculty-selected journal articles on the topic with student-led presentation and analysis of the articles. Students participate in practical scanning experiences during weekly Intensive Care Unit and Emergency Department ultrasound rounds. Students are evaluated through monthly quizzes and final examinations with mandatory requirements for logged ultrasound examinations in each ultrasound focus. Furthermore, assigned lectures and readings are provided through the online resource EMSono and previously developed YouTube presentations from OSU COM faculty.

\section{Ethical approval}

Ethical approval was obtained from The Ohio State University Office of Responsible Research Practices and Institutional Review Board. The study was deemed exempt research and a waiver of informed consent was approved by the board for the report.

\section{Results and discussion}

The major challenge to technology-focused curricular components generally lies with funding. Specific to focused ultrasonography, start-up costs include obtaining portable or handheld ultrasound machines and associated maintenance costs. Current models for portable ultrasound machines can range up to US\$60,000 per machine including service agreements and appropriate transducers. Handheld ultrasound machines may represent a drastically less expensive, although currently limited technologically option, with some machines ranging near US $\$ 10,000$ including service agreements. To limit equipment costs, this program utilized shared equipment with the medical center and relies upon clinical ultrasound machines for a large portion of the educational experience. Faculty time requirements were limited by designating a single faculty member as the ultrasound program director. Additional OSU COM faculty from departments with established ultrasound practices and experience were used to further enhance the program such as Emergency Medicine, Obstetrics and Gynecology, Radiology, Surgery, and Internal Medicine.

An additional issue facing new curricula is student time constraints and integration into existing teaching methodologies. Ultrasound has proven benefit as a teaching aid in anatomy, physiology, and clinical diagnosis and treatment $[15,18,19,23]$. Focused ultrasonography is a relatively novel teaching aid, and many faculty, even at academic centers, may not be proficient with the technology. The purpose of this program was to demonstrate that with limited faculty experienced in focused ultrasonography, a robust and vertical ultrasound curriculum can be created. Further, this curriculum has the potential to serve as a blueprint for other medical schools seeking to develop vertical ultrasound curricula. Focused ultrasound has been shown to have rapid uptake in basic skills and understanding, with learning being demonstrated in less than 1 day of lecture in some studies $[8,35]$. These aspects of focused ultrasound make the addition of ultrasound into pre-clinical and clinical medical student education potentially more feasible than other technologies which require large faculty teaching time and limited scope in medical education.

\section{Program improvement}

Components of the vertical ultrasound curriculum are evaluated by students using a survey before and after each required or elective component. Additionally, online quizzes have been introduced which evaluate student background knowledge and knowledge gained from each curricular component. A standardized method for assessing focused ultrasound knowledge however does not exist. Furthermore, there is no standardized instrument to assess focused ultrasound examination performance. A previously described scale developed at The Ohio State University College of Medicine, B-QUIET, is a reliable scale for assessing focused ultrasound image quality and is used for many of the course components [36]. Further research is needed to develop these methods for student evaluation and program efficacy.

As with all technologic advances, the medical education paradigm is challenged to develop an appropriate training regimen that integrates the information necessary for future physicians. While efforts to date have focused on graduate medical education for focused ultrasound training, numerous successful attempts to utilize focused ultrasonography exist at the medical school training level. The vertical ultrasound curriculum developed at The Ohio State University College of Medicine is a novel attempt in developing a vertical curriculum for medical school-level focused ultrasound training. In a future publication, the authors hope to demonstrate the impact of the vertical ultrasound curriculum on student clinical performance and advances made in the curriculum with experience.

\section{Conclusions}

Focused ultrasonography is an advancing evaluation and management application within medicine that assists practitioners in a rapid, noninvasive, and safe manner [1]. Evidence supporting focused ultrasonography is proliferating, and in conjunction, indications for its use are expanding. However, an ideal training program has yet to be developed despite a growing utilization of this 
technology. The vertical curriculum in ultrasound is an important initial effort to develop an evidenced-based training regimen at the medical school level, integrating focused ultrasonography as both a teaching adjunct and a clinical aid in training future physicians.

\section{Abbreviations}

ACEP: American College of Emergency Physicians; AMA: American Medical Association; CPT: Current Procedural Terminology; FAST: Focused Assessment with Sonography for Trauma; OSU COM: The Ohio State University College of Medicine.

\section{Competing interests}

The authors declare that they have no competing interests.

\section{Authors' contributions}

DPB conceived the vertical curriculum. DPB, EJA, and CTB contributed significantly to the design and implementation of the curriculum. NAR, DH, and $\mathrm{MB}$ performed the acquisition of data, analysis and interpretation of data, and initial manuscript preparation. All authors performed critical manuscript revisions. All authors read and approved the final manuscript.

\section{Authors' information}

DPB is a Professor of Emergency Medicine, the Director of Ultrasound in Emergency Medicine, and the Emergency Medicine Ultrasound Fellowship Director in the Department of Emergency Medicine, The Ohio State University College of Medicine. EJA is an assistant professor in the Department of Emergency Medicine with joint appointment with the Division of Pulmonary, Allergy, Critical Care, and Sleep Medicine in the Department of Internal Medicine, The Ohio State University College of Medicine. CTB is an assistant professor in the Department of Emergency Medicine, The Ohio State University College of Medicine. DH and MB are residents in the Department of Emergency Medicine, The Ohio State University College of Medicine. NAR is a resident in the Department of Surgery, Orlando Health, with joint appointment as a resident instructor at the University of Central Florida College of Medicine.

\section{Acknowledgements}

The authors wish to thank Dr. Daniel Clinchot for his work as Associate Dean for Medical Education at The Ohio State University College of Medicine. The authors also wish to thank Sharon Pfeil, Stacy Baker, and Sara Pastore for the administrative efforts associated with the ultrasound curriculum.

\section{Author details}

${ }^{1}$ Department of Emergency Medicine, The Ohio State University College of Medicine, 750 Prior Hall, 376 W 10th Avenue, Columbus, OH 43210, USA. 2Division of Pulmonary, Allergy, Critical Care, and Sleep Medicine, Department of Internal Medicine, The Ohio State University College of Medicine, 750 Prior Hall, 376 W 10th Avenue, Columbus, OH 43210, USA. ${ }^{3}$ Department of Surgery, Orlando Health, Orlando, FL 32806, USA. ${ }^{4}$ University of Central Florida College of Medicine, Orlando, FL 32827, USA.

Received: 21 September 2012 Accepted: 31 January 2013 Published: 2 July 2013

\section{References}

1. Moore CL, Copel JA (2011) Point-of-care ultrasonography. N Engl J Med 364 (8):749-757

2. Maitino AJ, Levin DC, Rao VM, Parker L, Sunshine JH (2005) Do emergency medicine physicians perform ultrasound and conventional radiography in the emergency department? Recent trends from 1993 to 2001. J Am Coll Radiol 2(3):274-278

3. American Medical Association House of Delegates (1999) Privileging for ultrasound imaging. H-230.960 (Res. 802) (I-99). American Medical Association, Chicago

4. American Medical Association House of Delegates (2000) Reimbursement for office-based or outpatient ultrasound imaging. H-385.934 (Sub. Res. 108) (A-00). American Medical Association, Chicago

5. American College of Emergency Physicians (2001) Use of ultrasound imaging by emergency physicians. Ann Emerg Med 38(4):469-470
6. American College of Emergency Physicians (2009) Emergency ultrasound guidelines. Ann Emerg Med 53(4):550-570

7. American Medical Association (2011) Physicians' Current Procedural Terminology (CPT) 2011. American Medical Association, Chicago

8. Thomas B, Falcone RE, Vasquez D, Santanello S, Townsend M, Hockenberry S, Innes J, Wanamaker S (1997) Ultrasound evaluation of blunt abdominal trauma: program implementation, initial experience, and learning curve. J Trauma 42(3):384-388. discussion 388-90

9. Accreditation Council for Graduate Medical Education (ACGME) (2012) Emergency Medicine Guidelines. http://www.acgme.org/acgmeweb/tabid/ 292/ProgramandInstitutionalGuidelines/Hospital-BasedAccreditation/ EmergencyMedicine/EmergencyMedicineGuidelines.aspx. Accessed February 2013

10. Accreditation Council for Graduate Medical Education (ACGME) (2008) ACGME Program Requirements for Graduate Medical Education in Obstetrics and Gynecology. http://www.acgme.org/acWebsite/downloads/ RRC_progReq/220obstetricsandgynecology01012008.pdf. Accessed February 2013

11. Accreditation Council for Graduate Medical Education (ACGME) (2008) Program Requirements for Graduate Medical Education in Diagnostic Radiology. https://www.acgme.org/acgmeweb/Portals/0/PFAssets/ ProgramRequirements/420_diagnostic_radiology_07012008.pdf. Accessed February 2013

12. Filly RA (1989) Radiology residency training in diagnostic sonography. J Ultrasound Med 8(9):475

13. Kern SJ, Smith RS, Fry WR, Helmer SD, Reed JA, Chang FC (1997) Sonographic examination of abdominal trauma by senior surgical residents. Am Surg 63(8):669-674

14. Akhtar S, Theodoro D, Gaspari R, Tayal V, Sierzenski P, Lamantia J, Stahmer S, Raio C (2009) Resident training in emergency ultrasound: consensus recommendations from the 2008 Council of Emergency Medicine Residency Directors Conference. Acad Emerg Med 16(Suppl 2):S32-S36

15. Wittich CM, Montgomery SC, Neben MA, Palmer BA, Callahan MJ, Seward JB, Pawlina W, Bruce CJ (2002) Teaching cardiovascular anatomy to medical students by using a handheld ultrasound device. JAMA 288(9):1062-1063

16. Shapiro RS, Ko PK, Jacobson S (2002) A pilot project to study the use of ultrasonography for teaching physical examination to medical students. Comput Biol Med 32(6):403-409

17. Yoo MC, Villegas L, Jones DB (2004) Basic ultrasound curriculum for medical students: validation of content and phantom. J Laparoendosc Adv Surg Tech A 14(6):374-379

18. Tshibwabwa ET, Groves HM (2005) Integration of ultrasound in the education programme in anatomy. Med Educ 39(11):1148

19. Decara JM, Kirkpatrick JN, Spencer KT, Ward RP, Kasza K, Furlong K, Lang RM (2005) Use of hand-carried ultrasound devices to augment the accuracy of medical student bedside cardiac diagnoses. J Am Soc Echocardiogr 18(3):257-263

20. Arger PH, Schultz SM, Sehgal CM, Cary TW, Aronchick J (2005) Teaching medical students diagnostic sonography. J Ultrasound Med 24(10):1365-1369

21. Hoppmann R, Cook T, Hunt P, Fowler S, Paulman L, Wells J, Richeson N, Thomas L, Wilson B, Neuffer F, McCallum J, Smith S (2006) Ultrasound in medical education: a vertical curriculum at the University of South Carolina School of Medicine. J S C Med Assoc 102(10):330-334

22. Tshibwabwa ET, Groves HM, Levine MA (2007) Teaching musculoskeletal ultrasound in the undergraduate medical curriculum. Med Educ 41(5):517-518

23. Butter J, Grant TH, Egan M, Kaye M, Wayne DB, Carrión-Carire V, McGaghie WC (2007) Does ultrasound training boost year 1 medical student competence and confidence when learning abdominal examination? Med Educ 41(9):843-848

24. Angtuaco TL, Hopkins RH, DuBose TJ, Bursac Z, Angtuaco MJ, Ferris EJ (2007) Sonographic physical diagnosis 101: teaching senior medical students basic ultrasound scanning skills using a compact ultrasound system. Ultrasound Q 23(2):157-160

25. Wright SA, Bell AL (2008) Enhancement of undergraduate rheumatology teaching through the use of musculoskeletal ultrasound. Rheumatology (Oxford) 47(10):1564-1566

26. Syperda VA, Trivedi PN, Melo LC, Freeman ML, Ledermann EJ, Smith TM, Alben JO (2008) Ultrasonography in preclinical education: a pilot study. J Am Osteopath Assoc 108(10):601-605 
27. Afonso N, Amponsah D, Yang J, Mendez J, Bridge P, Hays G, Baliga S, Crist K, Brennan S, Jackson M, Dulchavsky S (2010) Adding new tools to the black bag-introduction of ultrasound into the physical diagnosis course. J Gen Intern Med 25(11):1248-1252

28. Hoppmann RA, Rao W, Poston MB, Howe DB, Hunt PS, Fowler SD, Paulman LE, Wells JR, Richeson NA, Catalana PV, Thomas LK, Britt Wilson L, Cook T, Riffle S, Neuffer FH, McCallum JB, Keisler BD, Brown RS, Gregg AR, Sims KM, Powell CK, Garber MD, Morrison JE, Owens WB, Carnevale KA, Jennings WR, Fletcher S (2011) An integrated ultrasound curriculum (iUSC) for medical students: 4-year experience. Crit Ultrasound J 3(1):1-12

29. Rao S, van Holsbeeck L, Musial JL, Parker A, Bouffard JA, Bridge P, Jackson M, Dulchavsky SA (2008) A pilot study of comprehensive ultrasound education at the Wayne State University School of Medicine: a pioneer year review. J Ultrasound Med 27(5):745-749

30. Cook T, Hunt P, Hoppman R (2007) Emergency medicine leads the way for training medical students in clinician-based ultrasound: a radical paradigm shift in patient imaging. Acad Emerg Med 14(6):558-561

31. Kessler C, Bhandarkar S (2010) Ultrasound training for medical students and internal medicine residents—a needs assessment. J Clin Ultrasound 38(8):401-408

32. Bahner DP (2002) Trinity: a hypotensive ultrasound protocol. JDMS 18(4):193-198

33. Bahner DP, Hughes D, Royall NA (2012) I-AlM: a novel model for teaching and performing focused ultrasound. J Ultrasound Med 31(2):295-300

34. Blankenship R, Jones R (2011) EMSONO. http://www.emsono.com. Accessed February 2011

35. Frezza EE, Solis RL, Silich RJ, Spence RK, Martin M (1999) Competency-based instruction to improve the surgical resident technique and accuracy of the trauma ultrasound. Am Surg 65(9):884-888

36. Bahner DP, Adkins EJ, Nagel N, Way D, Werman HA, Royall NA (2011) Brightness mode quality ultrasound imaging examination technique (BQUIET): quantifying quality in ultrasound imaging. J Ultrasound Med 30(12):1649-1655

doi:10.1186/2036-7902-5-6

Cite this article as: Bahner et al.: Integrated medical school ultrasound: development of an ultrasound vertical curriculum. Critical Ultrasound Journal 2013 5:6.

\section{Submit your manuscript to a SpringerOpen ${ }^{\odot}$ journal and benefit from:}

- Convenient online submission

- Rigorous peer review

- Immediate publication on acceptance

- Open access: articles freely available online

- High visibility within the field

- Retaining the copyright to your article

Submit your next manuscript at $>$ springeropen.com 